\title{
AMENDMENTS
}

\section{Author Correction: Silicon-plasmonic integrated circuits for terahertz signal generation and coherent detection}

T. Harter (D), S. Muehlbrandt, S. Ummethala, A. Schmid, S. Nellen, L. Hahn D, W. Freude and C. Koos (D)

Correction to: Nature Photonics https://doi.org/10.1038/s41566-018-0237-x, published online 24 September 2018.

In the version of this Article originally published, on the left $y$ axis in Fig. 3c the label '50' was incorrect; it should have been ' 30 '. This has now been corrected in the online versions.

Published online: 11 June 2019

https://doi.org/10.1038/s41566-019-0487-2

\section{Publisher Correction: High-sensitivity imaging of time-domain near-infrared light transducer}

Yuyang Gu(D), Zhiyong Guo, Wei Yuan, Mengya Kong, Yulai Liu, Yongtao Liu (D), Yilin Gao, Wei Feng (DD, Fan Wang (DD, Jiajia Zhou (D), Dayong Jin (D) and Fuyou Li (D)

Correction to: Nature Photonics https://doi.org/10.1038/s41566-019-0437-z, published online 20 May 2019.

In the version of this Letter originally published online, in the sentence beginning "Here, we report a time-domain $(\tau)$ scheme", ' $>5,000$ ytterbium signal transducers' should have read 'about 5,000 ytterbium signal transducers'. And in the graph labelled 'Downshifting' in Fig. 1d the red peak was labelled 'Em.'; instead it should have been labelled 'Ex.' These errors have now been corrected in all versions.

Published online: 19 June 2019

https://doi.org/10.1038/s41566-019-0491-6 